\title{
Effect of Farnesol on Structure and Composition of Staphylococcus epidermidis Biofilm Matrix
}

\author{
Fernanda Gomes $\cdot$ Pilar Teixeira $\cdot$ Nuno Cerca $\cdot$ \\ Joana Azeredo • Rosário Oliveira
}

Received: 11 May 2011/Accepted: 1 July 2011 / Published online: 29 July 2011

(C) Springer Science+Business Media, LLC 2011

\begin{abstract}
Staphylococcus epidermidis is the most frequent cause of nosocomial sepsis and catheter-related infections in which biofilm formation is considered to be one of the main virulence mechanisms. Moreover, their increased resistance to conventional antibiotic therapy enhances the need to develop new therapeutical agents. Farnesol, a natural sesquiterpenoid present in many essential oils, has been described as impairing bacterial growth. The aim of this study was to evaluate the effect of farnesol on the structure and composition of biofilm matrix of $S$. epidermidis. Biofilms formed in the presence of farnesol $(300 \mu \mathrm{M})$ contained less biomass, and displayed notable changes in the composition of the biofilm matrix. Changes in the spacial structure were also verified by confocal scanning laser microscopy (CSLM). The results obtained by the quantification of extracellular polymers and by wheat germ agglutinin (WGA) fluorescent detection of glycoproteins containing $\beta(1 \rightarrow 4)-N$-acetyl-D-glucosamine support the hypothesis that farnesol causes disruption of the cytoplasmic membrane and consequently release of cellular content.
\end{abstract}

\section{Introduction}

Staphylococcus epidermidis is a coagulase-negative staphylococcus, which has emerged in the recent years as one of the most important nosocomial and opportunistic

F. Gomes · P. Teixeira · N. Cerca · J. Azeredo ·

R. Oliveira $(\square)$

Institute for Biotechnology and Bioengineering (IBB),

Centre of Biological Engineering, University of Minho,

Campus de Gualtar, 4710-057 Braga, Portugal

e-mail: roliveira@deb.uminho.pt pathogens [17]. Owing to its ability to attach to polymeric surfaces, $S$. epidermidis is a common pathogen in chronic medical device-associated infections [9, 11]. Nowadays, it is established that the natural mode of bacterial life is in multicellular complexes referred to as biofilms [16]. A biofilm can be defined as a surface-attached agglomeration of cells that are embedded in a heterogeneous matrix secreted by themselves $[19,20]$.

The matrix is one of the most distinctive features of a microbial biofilm. The composition of the matrix varies according to the nature of the organisms present. In general, the $S$. epidermidis biofilm matrix comprises several extracellular polymeric substances, such as polysaccharides, proteins, considerable amounts of extracellular teichoic acids and also extracellular DNA [17]. Two of the best characterized matrix polysaccharides in bacteria are alginate produced by Pseudomonas aeruginosa, and poly $\beta$-1,6-linked $N$-acetylglucosamine (PNAG) secreted by S. epidermidis. Synthesis of both polysaccharides has been related to bacterial virulence [1]. Essential for S. epidermidis cell accumulation is the expression of PNAG, also called polysaccharide intercellular adhesin (PIA), which mediates cell-to-cell adhesion [11, 17].

Bacterial pathogens have evolved numerous defence mechanisms against antimicrobial agents, and resistance to older and newly developed drugs is on the rise. Moreover, microbial biofilms being responsible for a number of diseases of chronic nature demonstrate extremely high resistance to antibiotics and host defence mechanisms [12, 18]. Farnesol, a natural sesquiterpenoid present in many essential oils, has been described to have antibacterial effect. Some authors have shown the antimicrobial effect of farnesol on S. epidermidis [7] and S. aureus [10] and it has been hypothesized that its mode of action is by compromising cell membrane integrity. Relatively to S. epidermidis, Gomes 
et al. (2009) demonstrated a high antimicrobial effect of farnesol against planktonic cells and a similar or higher effect than vancomycin against biofilm cells [8]. Since farnesol was defined as a potential alternative to antibiotics, namely those traditionally used to treat $S$. epidermidisrelated infections, such as vancomycin, it seems to be relevant to study and to try to understand the mechanism of action of this antimicrobial agent on $S$. epidermidis.

Because we are generally concerned with $S$. epidermidis biofilm control, the main aim of this study was to evaluate the effect of farnesol on biofilm structure and matrix composition.

\section{Materials and Methods}

\section{Bacterial Strains and Growth Conditions}

In this study, a good biofilm-producing strain was used, S. epidermidis 1457 . This strain is a clinical isolate and has been previously used in mutagenesis studies to determine the basis of biofilm formation [13]. Tryptic soy broth (TSB) and tryptic soy agar (TSA) were prepared according to the manufacturer's instructions. Strains were grown as previously described [2]. In brief, the strain was inoculated into $15 \mathrm{ml}$ of TSB from TSA plates not older than 2 days and grown for $18( \pm 2) \mathrm{h}$ at $37^{\circ} \mathrm{C}$ in an orbital shaker at $130 \mathrm{rpm}$. Cells were harvested by centrifugation (for $10 \mathrm{~min}$ at $9,500 \times g$ and $4^{\circ} \mathrm{C}$ ), and resuspended in TSB adjusted to an optical density $(640 \mathrm{~nm})$ equivalent to $1 \times 10^{9}$ cells ml $\mathrm{ml}^{-1}$ and then used in the subsequent assays. Each stock solution of farnesol was prepared in methanol. It was confirmed that methanol, at the concentration used, had no effect on the growth of the S. epidermidis strain studied.

\section{Biofilm Matrix Extraction}

Biofims were formed in six well tissue culture plates containing $4 \mathrm{ml}$ of $S$. epidermidis cell suspension $\left(1 \times 10^{6}\right.$ cells $\mathrm{ml}^{-1}$ ) in TSB supplemented with $0.25 \%$ glucose per well to promote biofilm formation. Plates were incubated at $37^{\circ} \mathrm{C}$ with orbital shaking at $130 \mathrm{rpm}$ for $24 \mathrm{~h}$. At the end, planktonic cells were removed carefully, and the biofilm was washed twice with $4 \mathrm{ml}$ of $0.9 \% \mathrm{NaCl}$. The biofilms were incubated in fresh nutrient medium containing farnesol (0, 30 and $300 \mu \mathrm{M})$ (Sigma) for $24 \mathrm{~h}$.

The extraction of the biofilm extracellular material was performed using the cation exchange Dowex resin $(50 \times 8$, $\mathrm{Na}^{+}$form, 20-50 mesh aldrich-fluka 44445), according to the procedure described by Frølund et al. [6]. Before extraction, the Dowex resin was washed with the extraction buffer $\left[2 \mathrm{mM} \mathrm{Na}_{3} \mathrm{PO}_{4} ; 4 \mathrm{mM} \mathrm{NaH} \mathrm{PO}_{4} ; 9 \mathrm{mM} \mathrm{NaCl}\right.$ and
$1 \mathrm{mM} \mathrm{KCl} ; \mathrm{pH}$ 7.0]. Then, the biofilms previously scrapped off the six well plates were washed with phosphate buffer (0.01 M; pH 7.0) and centrifuged for $5 \mathrm{~min}$, at $9,000 \times g$. The extraction was performed using $2 \mathrm{~g}$ of washed Dowex resin and $10 \mathrm{ml}$ of extraction buffer per gram of biofilm and stirring for $2 \mathrm{~h}$ at $400 \mathrm{rpm}$ and $-4^{\circ} \mathrm{C}$. The extracellular polymers (supernatant) were obtained by centrifugation at $9,000 \times g$ for $20 \mathrm{~min}$.

\section{Proteins and Polysaccharides Quantification}

The total protein content extracted from the matrix was determined by the colorimetric Bicinchoninic acid assay (Bicinchoninic Acid Kit for Protein Determination, Sigma, USA) using bovine serum albumin (BSA) as standard. The extracted polysaccharides were quantified by the phenolsulphuric acid method of Dubois et al. [5], using glucose as standard. All experiments were carried out in triplicate and repeated three times.

\section{Biofilm Dry-weight Measurements}

After $24 \mathrm{~h}$ of farnesol exposure, biofilm dry-weight was assessed. The biofilm cells were filtered through preweighed filters $(0.22 \mu \mathrm{m})$ and washed three times with ultrapure sterilized water. Filters were dried at $80^{\circ} \mathrm{C}$ until constant weight and cell dry weight were determined. This step was repeated at least four times. Biofilm dry-weights were assessed by the difference between the weight of the membrane with and without biomass.

\section{Confocal Scanning Laser Microscopy}

CSLM was performed as described before [3]. Biofilms were formed as described previously. Thereafter, farnesol at concentrations of 0 and $300 \mu \mathrm{M}$ was added to the preformed biofilms. Plates were incubated $24 \mathrm{~h}$ at $37^{\circ} \mathrm{C}$ and at $130 \mathrm{rpm}$. Following incubation, the biofilms were washed twice with $0.9 \% \mathrm{NaCl}$ and stained with DAPI and WGA (conjugated with Alexa Fluor 488-Molecular Probes) for the fluorescent detection of glycoproteins containing $\beta(1 \rightarrow 4)-N$-acetyl-D-glucosamine, or with live/dead staining, to determine cell viability. For live/dead staining, a negative control was employed to determine the baseline threshold for dead cells, by killing the biofilm with $96 \%$ ethanol for $4 \mathrm{~h}$. The plates were incubated for $20 \mathrm{~min}$ at room temperature in the dark. After staining, the biofilms were gently rinsed with $0.9 \% \mathrm{NaCl}$. The biofilm images $(1024 \times 1024)$ were acquired in an Olympus ${ }^{\text {TM }}$ FluoView FV1000 confocal scanning laser microscope. Biofilms were observed using a $60 \times$ water-immersion objective $(60 \times / 1.2 \mathrm{~W})$. For each condition, three independent biofilms were used, and in each biofilm four different regions 
of the surface were analysed. For biofilm maximum thickness determination, 20 different regions per surface were analysed, by determining the first and last layers of the biofilm, and calculating the maximum thickness of each region.

\section{Acquisition of Resistance/tolerance Determination}

S. epidermidis were grown planktonically in TSB with subinhibitory concentration of farnesol $(30 \mu \mathrm{M})$ for $12 \mathrm{~h}$. Then, the cells were harvested by centrifugation and adjusted to a cellular concentration of $\sim 1 \times 10^{6}$ cells $\mathrm{ml}^{-1}$ in fresh medium with and without farnesol (at an inhibitory concentration of $100 \mu \mathrm{M}$ ) for $24 \mathrm{~h}$ at $37^{\circ} \mathrm{C}$ at $130 \mathrm{rpm}$. Afterwards, cellular activity was assessed by colony-forming units (CFU). The initial cells, harvested after being in contact with a sub-inhibitory concentration of farnesol $(30 \mu \mathrm{M})$, were again subjected to that sub-inhibitory concentration of farnesol and this process was repeated for five consecutive days. Controls were cells not exposed to farnesol.

All the experiments were carried out in triplicate and repeated three times.

\section{Statistical Analysis}

The data from all assays were compared using one-way analysis of variance (ANOVA) by applying Tukey's and Bonferroni tests with all calculations carried out using SPSS software (Statistical Package for the Social Sciences). Differences achieving a confidence level of $95 \%$ were considered significant.

\section{Results}

Fig. 1a presents the quantification of polysaccharides and proteins in the biofilm matrix of S. epidermidis 1457 strain. According to the results of this study, after $24 \mathrm{~h}$ of farnesol $(300 \mu \mathrm{M})$ exposure, there was an increase in the exopolymers concentration present in the matrix of the biofilm $(P<0.05)$. On the other hand, the sub-inhibitory concentration of farnesol tested $(30 \mu \mathrm{M})$ appears to inhibit the formation of biofilm matrix as there was a reduction in the amount of existing exopolymers in the matrix $(P<0.05)$. Farnesol at $300 \mu \mathrm{M}$ caused a slight reduction on total biomass of biofilms of S. epidermidis 1457 strain (Fig. 1b).

WGA was employed to detect the presence of PNAG/ PIA [3] because this lectin binds to the biofilm matrix of S. epidermidis [14], because of its ability to recognize the $N$-acetylglucosamine component of PNAG/PIA antigen, although it may recognize other components, such as peptidoglycan and teichoic acid, which also contain glucosamine and form part of biofilm matrix [4].
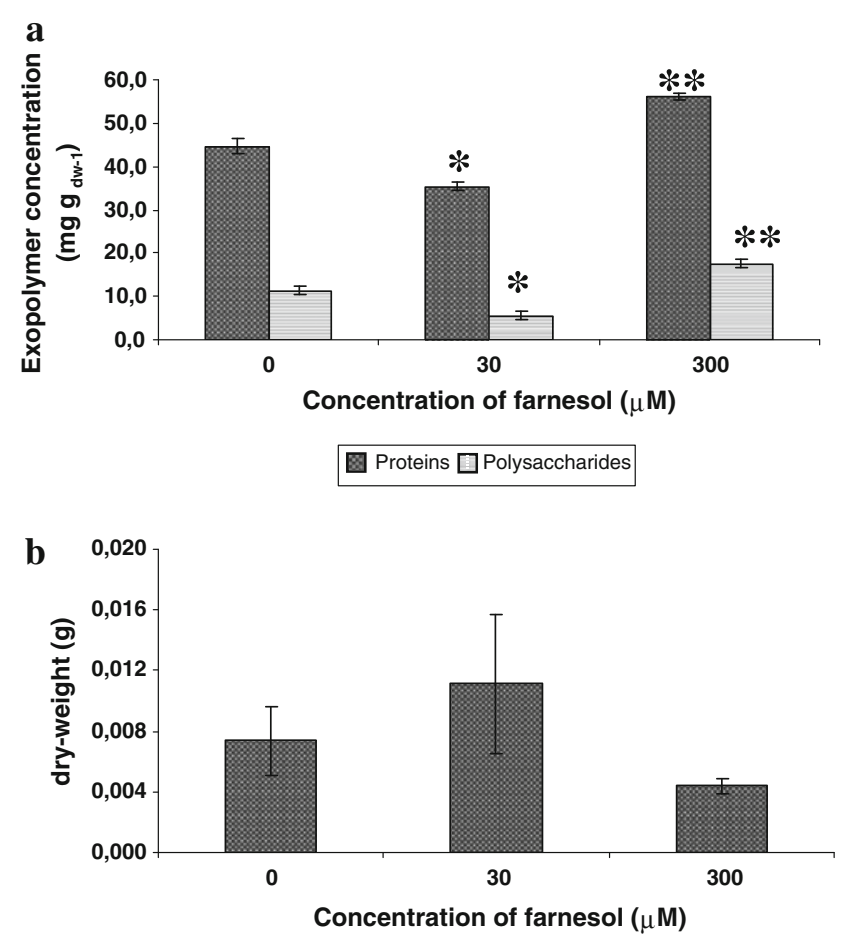

Fig. 1 Concentration of polysaccharides (glucose as standard) and proteins (BSA as standard) extracted $\left(\mathrm{mg}_{\mathrm{dw}-1}\right)$ by Dowex resin method from S. epidermidis strain 1457 biofilm matrix (a) and biofilm biomass expressed by dry weight (b). Error bars represent standard deviation. *Exopolymers concentration decreased significantly after treatment with $30 \mu \mathrm{M}$ farnesol compared with non-treated biofilms $(P<0.05)$; **Concentration of exopolymers after treatment with $300 \mu \mathrm{M}$ of farnesol significantly increased compared with non-treated biofilms $(P<0.05)$

CSLM images showed that S. epidermidis 1457 strain formed a thick biofilm when grown in the absence of farnesol having a noticeable amount of PNAG/PIA. After addition of farnesol, a significant destruction of biofilm structure (Fig. 2a) and a clear reduction of biofilm thickness (Fig. 3) were observed. Furthermore, most cells inside the biofilm were either dead or with damaged cell wall, as determined by live/dead staining (Fig. 2b).

Although the mechanism of action of farnesol is not yet understood, it appears to have an antimicrobial effect against $S$. epidermidis cells [7]. It was previously shown that sometimes biofilm bacteria can be induced to increase their resistance to antimicrobials by continuing exposure to sub-inhibitory concentrations of antibiotics [3]. Therefore, it is important to study the ability of cells to acquire tolerance/resistance to this agent. For this purpose, planktonic cells were treated with a sub-inhibitory concentration of farnesol over several generations to determine whether after contact with this compound, the cells acquire tolerance/resistance. The results obtained in this study are shown in Fig. 4. 

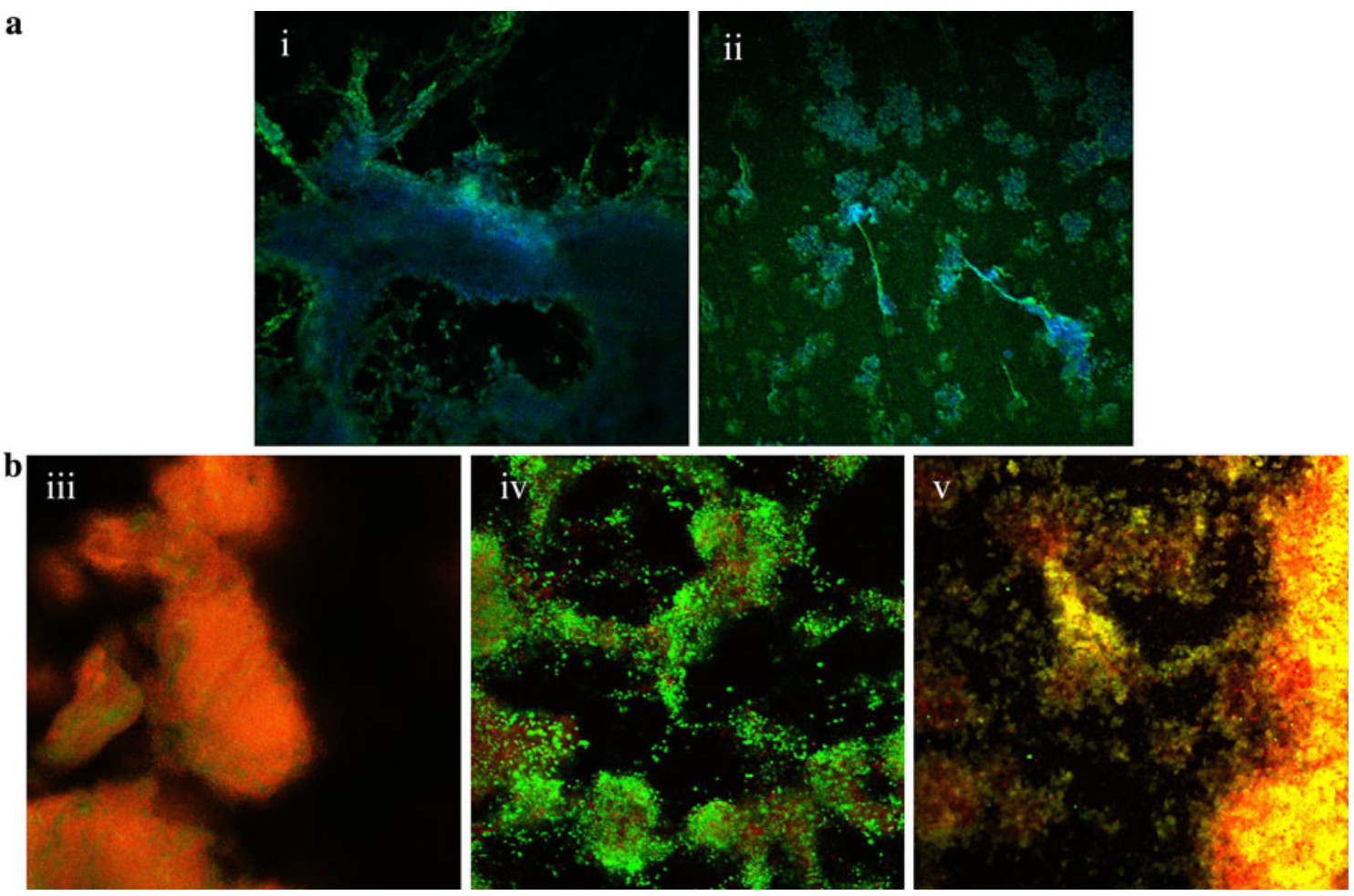

Fig. 2 a CLSM images of 24-h biofilm following 24-h exposure to $0 \mu \mathrm{M}($ i) and $300 \mu \mathrm{M}$ (ii) farnesol, stained with DAPI and WGA. b Biofilms stained with live/dead: (iii) left image is a negative control

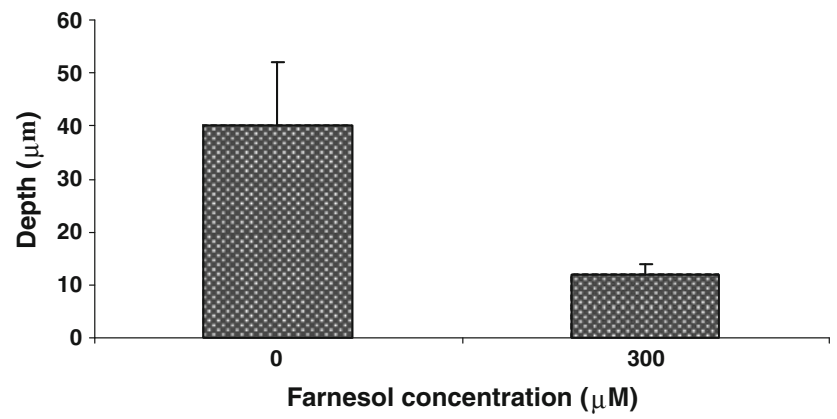

Fig. 3 Biofilm maximum depth average obtained by CSLM for biofilms without exposure to farnesol and after 24-h exposure to $300 \mu \mathrm{M}$ of farnesol. *Statistically different from control (untreated cells $)(P<0.05)$

The results of CFU determination evidenced an acquisition of tolerance to farnesol by the cells after being exposed to farnesol at a concentration of $30 \mu \mathrm{M}$. However, over time, no resistance to farnesol was observed.

\section{Discussion}

Nowadays, S. epidermidis ranks first amongst the causative agents of nosocomial infections and represents the most (killed biofilm bacteria killed with $96 \%$ ethanol), (iv) biofilm following 24-h exposure to $0 \mu \mathrm{M}$ farnesol and $(v)$ biofilm exposed for $24-\mathrm{h}$ to $300 \mu \mathrm{M}$ farnesol

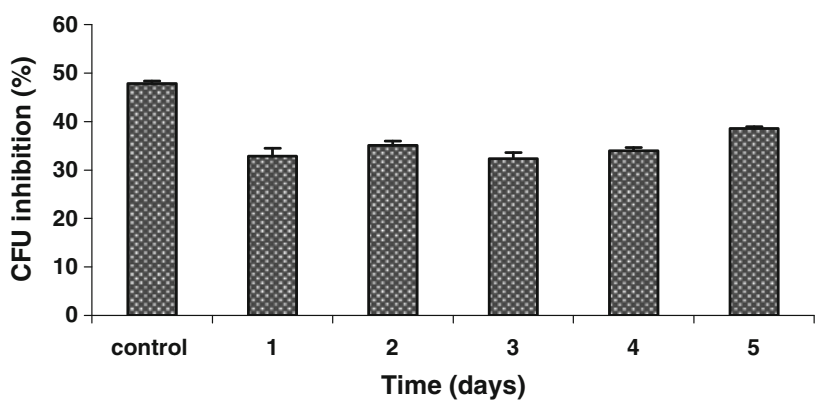

Fig. 4 Percentage of CFU inhibition by farnesol against planktonic $S$. epidermidis cells (strain 1457). Control corresponds to cells not exposed to sub-inhibitory concentrations of farnesol. Error bars represent standard deviation

common cause of infections on indwelling medical devices [15]. Simultaneously, the resistance to antibiotics has become an important problem in S. epidermidis infections. In this context, the interest in studying the antimicrobial activity of potential alternatives to antibiotics has increased in recent years. In a previous study, the effect of farnesol against biofilm cells of six clinical isolates of $S$. epidermidis strains was assessed after $24 \mathrm{~h}$ of exposure. The strains assayed were 1457, 9142, IE186, IE75, IE214 and LE7 [8], and no significant difference was observed in the effect of farnesol on biofilm cells of the different strains 
(some results not shown). However, as determined by Sousa et al. [17], S. epidermidis 1457 strain is the one, which has the highest level of extracellular polysaccharides content and, consequently, the strain that formed thicker biofilms. Taking this into consideration, we selected this strain as the most extreme example to evaluate the role of farnesol in biofilm structure and matrix composition.

First, the polysaccharide and protein biofilm matrix content, as well as the total biomass of biofilm were quantified. The results of this study showed an increase in proteins and polysaccharides per gram dry weight of biofilm after treatment with farnesol at $300 \mu \mathrm{M}$. This may be derived from the bursting of the cells and consequent release of cellular content. This fact can also be due to an overexpression of some $S$. epidermidis virulence genes responsible for the production of PNAG/PIA and other exopolymers, which can be a protective mechanism triggered by cells under stress. On the other hand, there is a slight decrease in the amount of polysaccharides and proteins in the extracellular matrix after exposure to $30 \mu \mathrm{M}$ of farnesol. This sub-inhibitory concentration has no effect either on cell metabolic activity and consequently in cell replication or in the total biofilm biomass (Fig. 1b) $(P>0.05)$ [7]. Thus, although this concentration was not significantly inhibitory against $S$. epidermidis biofilm, it can possibly decrease the matrix development and therefore the biofilm formation over time.

Many S. epidermidis strains produce exopolymers, namely poly-gama-glutanic acid and a PNAG homopolymer, also named PIA, which surrounds and connects $S$. epidermidis cells inside biofilms [15]. In general, S. epidermidis exopolymers protect the cells from antibody recognition and consequently protect the bacterium from important mechanisms of innate host defence. Relative to PNAG/PIA, in addition to its role as part of the extracellular biofilm matrix, it has been found to protect S. epidermidis from neutrophil killing, complement deposition, immunoglobulins and antimicrobial peptides (AMPs) $[4,15]$. Moreover, our immune system may have evolved to react less strongly to prevalent colonising bacteria, hampering $S$. epidermidis biofilm eradication.

The presence of PNAG/PIA in $S$. epidermidis biofilm was detected by WGA binding [3]. WGA is a carbohydrate-binding protein of approx. $36 \mathrm{kDa}$, which selectively recognizes sialic acid and $N$-acetylglucosaminyl sugar residues which are predominantly found in biofilm matrix and namely in PNAG/PIA molecules. Farnesol at $300 \mu \mathrm{M}$ promoted a modification in biofilm structure and a decrease in biofilm thickness. The decrease in biofilm thickness could either be a result of biofilm bacteria death or biofilm bacteria dispersion. To clarify this point, biofilms were stained with live/dead, and as indicated by these results the observed biofilm reduction was in fact mainly due to cell death (Fig. 2b). As we have previously shown S. epidermidis planktonic cells after $12-\mathrm{h}$ exposure to $300 \mu \mathrm{M}$ farnesol have an average reduction of about $4 \mathrm{log}$. Therefore, any cells released from 24-h-treated biofilms are most likely killed when assuming a planktonic lifestyle.

To test the potential antimicrobial action of farnesol against $S$. epidermidis, it is crucial to determine the cells ability to acquire tolerance/resistance. For this purpose, S. epidermidis cells were exposed to sub-inhibitory and inhibitory concentrations of farnesol.

Staphylococcus epidermidis 1457 seems to be capable of rapid adaptation after an initial contact with farnesol but this effect was not a progressive mechanism. Therefore, it can be concluded that this is a reversible mechanism and therefore, a case of tolerance and not resistance. Moreover, resistance is translated by an increased survival of individuals which are immune to the effects of the antibacterial agent, whose offsprings then inherit the resistance creating a new population of resistant bacteria. The results of this study demonstrated that over cell generations there is no decrease in the susceptibility of farnesol.

In conclusion, the results obtained by the quantification of extracellular polymers and by WGA fluorescent detection of PNAG/PIA support the hypothesis that farnesol causes disruption of the cytoplasmic membrane and consequently release of cellular content. In addition to cell death, farnesol seems to destroy the biofilm structure reducing its biomass. This general promotion of biofilm weakness may be a potential help for the human immune system to eradicate the focus of $S$. epidermidis infection.

Acknowledgment Fernanda Gomes and Pilar Teixeira fully acknowledge the financial support of Fundação para a Ciência e Tecnologia (FCT) through the grants SFRH/BD/32126/2006 and SFRH/BPD/26803/2006, respectively.

\section{References}

1. Al-Fattani MA, Douglas LJ (2006) Biofilm matrix of Candida albicans and Candida tropicalis: chemical composition and role in drug resistance. J Med Microbiol 55:999-1008

2. Cerca N, Pier GB, Oliveira R et al (2004) Comparative evaluation of coagulase-negative staphylococci (CoNS) adherence to acrylic by a static method and a parallel-plate flow dynamic method. Res Microbiol 155:755-760

3. Cerca N, Martins S, Sillankorva S et al (2005) Effects of growth in the presence of subinhibitory concentrations of dicloxacillin on Staphylococcus epidermidis and Staphylococcus haemolyticus biofilms. Appl Environ Microbiol 71:8677-8682

4. Cerca N, Jefferson KK, Oliveira R et al (2006) Comparative antibody-mediated phagocytosis of Staphylococcus epidermidis cells grown in a biofilm or in planktonic state. Infect Immun 74:4819-4855

5. Dubois M, Gilles KA, Hamilton JK et al (1956) Colorimetric method for determination of sugars and related substances. Anal Chem 28:350-356 
6. Frølund B, Palmgren R, Keiding K et al (1996) Extraction of extracellular polymers from activated sludge using a cation exchange resin. Water Res 30:1749-1758

7. Gomes FIA, Teixeira P, Azeredo J et al (2009) Effect of farnesol on planktonic and biofilm cells of Staphylococcus epidermidis. Curr Microbiol 59:118-122

8. Gomes F, Leite B, Teixeira P et al (2011) Farnesol as antibiotics adjuvant in Staphylococcus epidermidis control in vitro. Am J Med Sci 341:191-195

9. Izano EA, Sadovskaya I, Vinogradov E et al (2007) Poly- $N$ acetylglucosamine mediates biofilm formation and antibiotic resistance in Actinobacillus pleuropneumoniae. Microb Pathog 43:1-9

10. Jabra-Rizk MA, Meiller TF, James CE et al (2006) Effect of farnesol on Staphylococcus aureus biofilm formation and antimicrobial susceptibility. Antimicrob Agents Chemother 50:14631469

11. Knobloch JK, Osten HV, Horstkotte MA et al (2002) Minimal attachment killing (MAK): a versatile method for susceptibility testing of attached biofilm-positive and negative Staphylococcus epidermidis. Med Microbiol Immunol 191:107-114

12. Kuźma Ł, Różalski M, Walencka E et al (2007) Antimicrobial activity of diterpenoids from hairy roots of Salvia sclarea L.: salvipisone as a potential anti-biofilm agent active against antibiotic resistant Staphylococci. Phytomedicine 14:31-35
13. Mack D, Haeder M, Siemssen N et al (1996) Association of biofilm production of coagulase-negative staphylococci with expression of a specific polysaccharide intercellular adhesin. J Infect Dis 174:881-884

14. Neu T, Swerhone GD, Lawrence JR (2001) Assessment of lectinbinding analysis for in situ detection of glycoconjugates in biofilm systems. Microbiology 147:299-313

15. Otto M (2009) Staphylococcus epidermidis - the "accidental" pathogen. Microbiology 7:555-567

16. Sandberg M, Määttänen A, Peltonen J et al (2008) Automating a 96-well microtitre plate model for Staphylococcus aureus biofilms: an approach to screening of natural antimicrobial compounds. Int J Antimicrob Agents 32:233-240

17. Sousa C, Teixeira P, Oliveira R (2009) The role of extracellular polymers on Staphylococcus epidermidis biofilm biomass and metabolic activity. J Basic Microbiol 49:363-370

18. Teixeira PC, Leite GM, Domingues RJ et al (2007) Antimicrobial effects of a microemulsion and a nanoemulsion on enteric and other pathogens and biofilms. Int J Food Microbiol 118:15-19

19. Vuong C, Kocianova S, Yao Y et al (2004) Increased colonization of indwelling medical devices by quorum-sensing mutants of Staphylococcus epidermidis in vivo. J Infect Dis 190:1498-1505

20. Ziebuhr W, Hennig S, Eckart M et al (2006) Nosocomial infections by Staphylococcus epidermidis: how a commensal bacterium turns into a pathogen. Int J Antimicrob Agents 28S:S14-S20 